\title{
Study of shear viscosity of SU(2)-gluodynamics within lattice simulation
}

\author{
N.Yu. Astrakhantsev, ${ }^{a, b}$ V.V. Braguta ${ }^{a, c, d, e}$ and A.Yu. Kotov ${ }^{a, e}$ \\ ${ }^{a}$ Institute for Theoretical and Experimental Physics, \\ Moscow, 117218 Russia \\ ${ }^{b}$ Moscow Institute of Physics and Technology, \\ Dolgoprudny, 141700 Russia \\ ${ }^{c}$ Institute for High Energy Physics NRC "Kurchatov Institute", \\ Protvino, 142281 Russian Federation \\ ${ }^{d}$ Far Eastern Federal University, School of Biomedicine, \\ Vladivostok, 690950 Russia \\ ${ }^{e}$ National Research Nuclear University MEPhI (Moscow Engineering Physics Institute), \\ Kashirskoe highway, 31, Moscow, 115409 Russia \\ E-mail: nikita.astrakhantsev@itep.ru, braguta@itep.ru, kotov@itep.ru
}

ABSTRACT: This paper is devoted to the study of two-point correlation function of the energy-momentum tensor $\left\langle T_{12} T_{12}\right\rangle$ for $\mathrm{SU}(2)$-gluodynamics within lattice simulation of QCD. Using multilevel algorithm we carried out the measurement of the correlation function at the temperature $T / T_{c} \simeq 1.2$. It is shown that lattice data can be described by spectral functions which interpolate between hydrodynamics at low frequencies and asymptotic freedom at high frequencies. The results of the study of spectral functions allowed us to estimate the ratio of shear viscosity to the entropy density $\eta / s=0.134 \pm 0.057$.

KeYwords: Quark-Gluon Plasma, Lattice Gauge Field Theories

ARXiv EPRINT: 1507.06225 


\section{Contents}

1 Introduction 1

2 Details of the calculation $\quad 2$

3 Numerical results $\quad 4$

4 Conclusion $\quad 8$

\section{Introduction}

One of the most important result obtained at RHIC experiment is the measurement of the elliptic flow of final particles $[1,2]$. The value of the elliptic flow measured at RHIC can be explained within the approach based on hydrodynamics [3-5], if one assumes that quark-gluon plasma (QGP) obtained after the collision is almost superfluid. In particular, numerical simulations of the relativistic liquid showed [6] that the upper limit on the ratio of shear viscosity to entropy density is $\eta / s \leq 0.4$. Preferred range for the ratio is $\eta / s=(1 \leftrightarrow 3) \times 1 / 4 \pi$, which is very close to the result of $\mathrm{N}=4$ Super Yang Mills (SYM) theory at strong coupling $\eta / s=1 / 4 \pi$ [7]. From these facts one can conclude that theoretical prediction of the ratio $\eta / s$ is a very interesting and important problem.

It is known that QGP is strongly interacting system and today there are no analytical approaches which allow to study such systems without additional assumptions. For this reason one of the main approaches which can be used to study the properties of QGP and which is based on the first principles is lattice simulation of QCD.

Lattice simulation of QCD aimed at the calculation of shear viscosity of the SU(3)gluodynamics was carried out in papers [8-11]. Despite rather large uncertainties one can state that the ratios $\eta / s$ obtained in papers $[10,11]$ are close to the $N=4$ SYM prediction $\eta / s \sim 1 / 4 \pi$. The calculation of shear viscosity in QCD with dynamical quarks is still a challenging problem.

The closeness of the $\mathrm{SU}(3)$-gluodynamics ratio $\eta / s$ to the $\mathrm{N}=4 \mathrm{SYM}$ prediction allows us to ask the question whether this property is the property of the $\mathrm{SU}(3)$-gluodynamics or it is common to all non-abelian gauge theories. To address this question in this paper we are going to perform the calculation of shear viscosity of $\mathrm{SU}(2)$-gluodynamics within lattice simulation.

We have already performed the first measurement of shear viscosity of the $\mathrm{SU}(2)$ gluodynamics in [12]. In this paper we extend our investigation to larger lattice size and apply different method to determine numerical value of the ratio $\eta / s$. 


\section{Details of the calculation}

Shear viscosity is related to the Euclidean correlation function of the energy-momentum tensor $T_{\mu \nu}=\frac{1}{4} \delta_{\mu \nu} F_{\alpha \beta}^{a} F_{\alpha \beta}^{a}-F_{\mu \alpha}^{a} F_{\nu \alpha}^{a}$ (here we omitted for simplicity trace anomaly):

$$
C\left(x_{0}\right)=T^{-5} \int d^{3} \mathbf{x}\left\langle T_{12}(0) T_{12}\left(x_{0}, \mathbf{x}\right)\right\rangle,
$$

where $T$ is the temperature of the system. The correlation function (2.1) can be written in terms of the spectral function $\rho(\omega)$ as follows

$$
C\left(x_{0}\right)=T^{-5} \int_{0}^{\infty} \rho(\omega) \frac{\cosh \omega\left(\frac{1}{2 T}-x_{0}\right)}{\sinh \frac{\omega}{2 T}} d \omega .
$$

The spectral function contains a lot of important information about the properties of medium. In particular, to find shear viscosity from spectral function one uses the Kubo formula [13]

$$
\eta=\pi \lim _{\omega \rightarrow 0} \frac{\rho(\omega)}{\omega}
$$

Lattice calculation of shear viscosity can be divided into two parts. The first part is the measurement of the correlation function $C\left(x_{0}\right)$ with sufficient accuracy. This part of the calculation requires large computational resources but for the gluodynamics the accuracy of the correlator can be dramatically improved with the help of the two-level algorithm [14]. The second part is the determination of the spectral function $\rho(\omega)$ from the correlation function $C\left(x_{0}\right)$. The last part of the calculation is probably the most complicated, since one should determine continuous spectral function $\rho(\omega)$ from integral equation (2.2) for the set of $O(10)$ values of the function $C\left(x_{0}\right)$ measured in lattice simulation.

To approach the solution of the integral equation (2.2) one should take into account the properties of the spectral function. Important properties of the spectral function are positivity: $\rho(\omega) / \omega \geq 0$ and oddness: $\rho(-\omega)=-\rho(\omega)$. It is also important to write the expression for the spectral function at the leading-order approximation in strong coupling constant [15]

$$
\rho^{\mathrm{LO}}(\omega)=\frac{1}{10} \frac{d_{A}}{(4 \pi)^{2}} \frac{\omega^{4}}{\tanh \left(\frac{\omega}{4 T}\right)}+\left(\frac{2 \pi}{15}\right)^{2} d_{A} T^{4} \omega \delta(\omega),
$$

where $d_{A}=N_{c}^{2}-1=3$ for the $\mathrm{SU}(2)$-gluodynamics. It can be shown in the hard-thermalloop framework that the term proportional $\delta$-function is modified to some function of final width due to interactions [16]. For instance, one can approximate this function by $\delta(\omega) \sim 1 /\left(1+b^{2} \omega^{2}\right)$. However, below it will be shown that our lattice data don't support this form of the spectral function.

One also knows next-to-leading order expression for the spectral function at large $\omega[17]$

$$
\lim _{\omega \rightarrow \infty} \rho^{\mathrm{NLO}}(\omega)=\frac{1}{10} \frac{d_{A}}{(4 \pi)^{2}} \omega^{4}\left(1-\frac{5 \alpha_{s} N_{c}}{9 \pi}\right) .
$$

It should be noted here that at large $\omega$ the spectral function scales as $\rho(\omega) \sim \omega^{4}$, what leads to a large perturbative contribution to the correlation function for all values of Euclidean 
time $x_{0}$. Calculation shows that even at the $x_{0}=1 /(2 T)$ the tree level contribution is $\sim 85 \%$ of the total value of the correlation function. Note also that large $\omega$ behaviour of the spectral function leads to a fast decrease of the correlation function $C\left(x_{0}\right) \sim 1 / x_{0}^{5}$ for small $x_{0}$. For this reason the signal/noise ratio for the $C\left(x_{0}\right)$ is small at $x_{0} \gg a$ and lattice measurement of the correlation function at $x_{0} \sim 1 /(2 T)$ becomes computationally very expensive.

In numerical simulation we use Wilson gauge action for the $\mathrm{SU}(2)$-gluodynamics

$$
S_{g}=\beta \sum_{x, \mu<\nu}\left(1-\frac{1}{2} \operatorname{Tr} U_{\mu, \nu}(x)\right),
$$

where $U_{\mu, \nu}(x)$ is the product of the link variables along elementary rectangular $(\mu, \nu)$, which starts at $x$.

For the tensor $F_{\mu \nu}$ we use the clover discretization scheme:

$$
\begin{aligned}
F_{\mu \nu}^{(\text {clov })}(x) & =\frac{1}{4 i g a^{2}}\left(V_{\mu, \nu}(x)+V_{\nu,-\mu}(x)+V_{-\mu,-\nu}(x)+V_{-\nu, \mu}(x)\right) \\
V_{\mu, \nu}(x) & =\frac{1}{2}\left(U_{\mu, \nu}(x)-U_{\nu, \mu}(x)\right) .
\end{aligned}
$$

It causes no difficulties to build energy-momentum tensor having expression for the tensor $F_{\mu \nu}$.

To calculate shear viscosity one should measure the correlation function (2.1). To carry out this measurement we use two-level algorithm described in [14]. This algorithm significantly improves the speed of the calculations. Note also that instead of the correlation function $\left\langle T_{12}(x) T_{12}(y)\right\rangle$ in this paper we measure the correlation function $\frac{1}{2}\left(\left\langle T_{11}(x) T_{11}(y)\right\rangle-\left\langle T_{11}(x) T_{22}(y)\right\rangle\right)$. Both correlation functions are equal in the continuum limit $[8]$.

It has become conventional to present the value of shear viscosity as a the ratio viscosity-to-entropy density $\eta / s$. For homogeneous systems the entropy density $s$ can be expressed as $s=\frac{\epsilon+p}{T}$, where $\epsilon$ is the energy density and $p$ is the pressure. These thermodynamic quantities were measured with the method described in [18].

Energy-momentum tensor in continuum theory is a set of Noether currents which are related to the translation invariance of the action. In lattice formulation of field theory continuum translation invariance does not exist and renormalization for energy-momentum tensor is required. For the correlation function considered in this paper the renormalization is multiplicative [19]. Renormalization factors depend on the discretization scheme. For instance, for the diagonal component of $T_{\mu \nu}$ (when $\mu=\nu$ ) and the plaquette-based discretization of $T_{\mu \nu}: T_{\mu \mu}=\frac{2}{a^{4} g^{2}}\left(-\sum_{\nu \neq \mu} \operatorname{Tr} U_{\mu, \nu}(x)+\sum_{\nu, \sigma \neq \mu, \sigma>\nu} \operatorname{Tr} U_{\sigma, \nu}(x)\right)$ the renormalization factors are related to the anisotropy coefficients [20, 21]: $T_{\mu \nu}^{(\text {ren })}=Z^{\text {(plaq) }} T_{\mu \nu}^{(\text {plaq })}$, $Z^{\text {(plaq) }}=1-\frac{1}{2} g_{0}^{2}\left(c_{\sigma}-c_{\tau}\right)$, where $c_{\sigma}$ and $c_{\tau}$ are defined in [18] and for $\mathrm{SU}(2)$ are computed on the lattice in [22].

Using the renormalization factors for the plaquette-based discretization of $T_{00}$, we can find the renormalization factors for the clover discretization simply by fitting the vacuum expectation values of the renormalized $T_{00}: Z^{(\text {plaq })}\left\langle T_{00}^{(\text {plaq })}\right\rangle=Z^{(\text {(lov) }}\left\langle T_{00}^{(\text {clov })}\right\rangle$. 


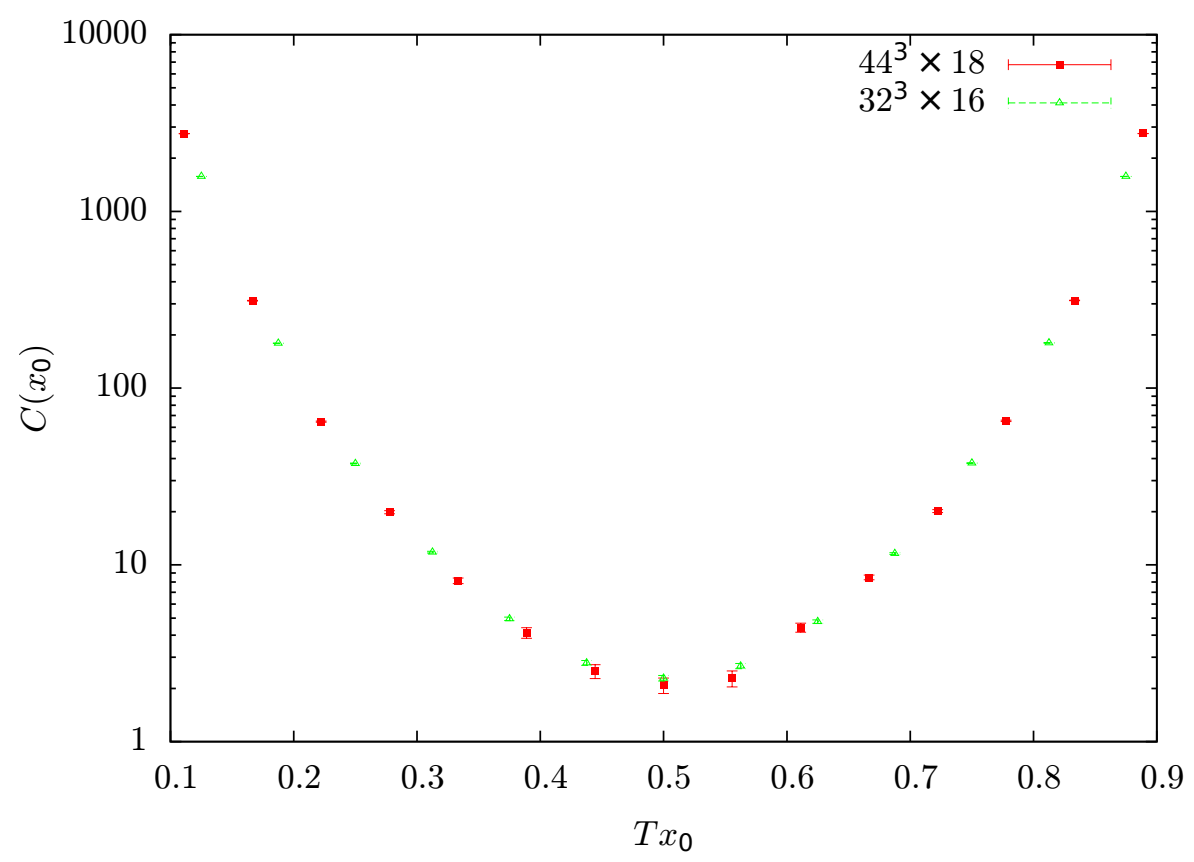

Figure 1. Renormalized correlation functions $C\left(x_{0}\right)$ as a function of Euclidean time $x_{0}$ measured at lattices $16 \times 32^{3}$ and $18 \times 44^{3}$.

\section{Numerical results}

We carried out the measurements of the correlation function $C\left(x_{0}\right)$ at lattices $16 \times 32^{3}$ with $\beta=2.81$ and $18 \times 44^{3}$ with $\beta=2.85$. Both lattices correspond to deconfinement phase with the temperature $T / T_{c} \simeq 1.2$ but different physical volumes and lattice spacings. In figure 1 we plot renormalized correlation functions (2.1) as a function of Euclidean time $x_{0}$ for the lattices $16 \times 32^{3}$ and $18 \times 44^{3}$. Two-level algorithm allowed us to reach accuracy $\sim 3 \%$ for the lattice $16 \times 32^{3}$ and $\sim 10 \%$ for the lattice $18 \times 44^{3}$ at point $T x_{0}=0.5$. For the other points the accuracy is much better.

Visually the set of values of the correlation functions measured at different lattices lies on one curve. The values for the correlation functions measured at close points in the region $T x_{0} \sim 0.5$ are very close to each other. Note also that the fit parameters (see below) of both correlation functions are very close to each other. These facts allow us to expect that finite lattice size and finite volume effects are not very important in our calculation.

Usually one uses Maximal Entropy Method to study transport properties of QGP (see, for instance, $[23,24])$. Unfortunately the accuracy of our results and number of points where the correlation function (2.1) was measured do not allow us to use Maximal Entropy Method to determine the spectral function $\rho(\omega)$. For this reason we use physically motivated ansatz for the spectral function with unknown parameters which will be determined through the fitting procedure. Probably the simplest formula for the spectral function inspired by QCD sum rules [25] can be built if we join hydrodynamical behaviour at small frequencies with asymptotic freedom at large frequencies ${ }^{1}$

$$
\rho_{1}(\omega)=B T^{3} \omega \theta\left(\omega_{0}-\omega\right)+A \rho_{\text {lat }}(\omega) \theta\left(\omega-\omega_{0}\right) .
$$

\footnotetext{
${ }^{1}$ Note that the frequency $\omega$ is measured in physical units.
} 


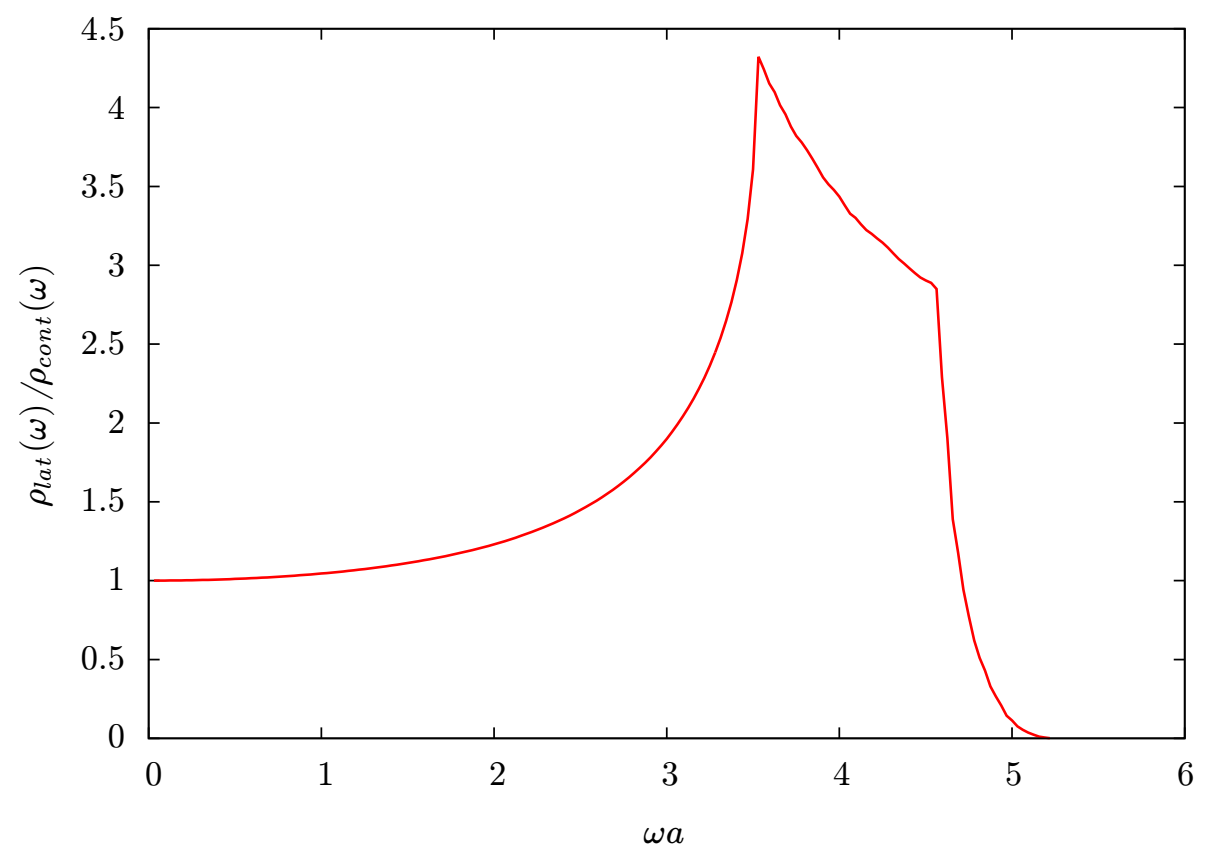

Figure 2. The ratio $\rho_{\text {lat }}(\omega) / \rho_{\text {cont }}(\omega)$ as a function of $\omega a$.

In last formula $\rho_{\text {lat }}(\omega)$ is a tree level lattice expression for the spectral function calculated for the correlation function $\sim \frac{1}{2}\left(\left\langle T_{11}(x) T_{11}(y)\right\rangle-\left\langle T_{11}(x) T_{22}(y)\right\rangle\right)$ with clover discretization of the tensor $F_{\mu \nu}$ at lattice with fixed $L_{t}$ and $L_{s} \rightarrow \infty$. Although the calculation of the $\rho_{\text {lat }}(\omega)$ can be easily performed using formulas from paper [26], the resulting expression is very cumbersome. For this reason instead of the explicit expression for the $\rho_{\text {lat }}$, in figure 2 we plot the ratio $\rho_{\text {lat }}(\omega) / \rho_{\text {cont }}(\omega)$ for the lattice $L_{t}=16$.

The fit of the lattice data $\left(x_{0} / a \geq 2\right)$ with the formula (2.2) with the spectral function (3.1) gives $A=0.723 \pm 0.002, B=0.079 \pm 0.016, \omega_{0} / T=7.5 \pm 0.5, \chi^{2} /$ dof $\simeq 1.4$ for the lattice $16 \times 32^{3}$ and $A=0.703 \pm 0.003, B=0.096 \pm 0.026, \omega_{0} / T=8.3 \pm 0.7, \chi^{2} / d o f \simeq 0.7$ for the lattice $18 \times 44^{3}$. It is seen that ansatz (3.1) for the spectral function fits the lattice data very well and the parameters of the fit for different lattices are in a reasonable agreement with each other. The resulting shear viscosity is $\eta / s=0.179 \pm 0.036$ for the lattice $16 \times 32^{3}$ and $\eta / s=0.217 \pm 0.059$ for the lattice $18 \times 44^{3}$. In figure 3 we plot the spectral function $\rho_{1}(\omega)$ as a function $\omega$ for the lattice $L_{t}=16$.

Note that in hard-thermal-loop framework hydrodynamical behaviour at small frequencies is replaced by transport peak of final width $\sim \omega \rightarrow \omega /\left(1+b^{2} \omega^{2}\right)[16]$. The $\chi^{2} /$ dof at lattice $16 \times 32^{3}$ for this form of the spectral function is minimized at $b \sim 0$ with the result $\eta / s=0.20 \pm 3$. Thus within the unsertainty of the calculation the transport peak is reduced to hydrodynamical form (3.1) with the same shear viscosity.

Now few comments are in order

- In formula (3.1) we used $\rho_{\text {lat }}(\omega)$ instead of the continuum tree-level expression for the $\rho(\omega)$ (see formula (2.4)). We believe that this allows us to take into account discretization uncertainty and asymptotic freedom contribution more carefully. If 
one puts the continuum tree-level expression to formula (3.1), the $\chi^{2} / d o f$ of the fits will be considerably enhanced: $\chi^{2} / d o f \simeq 5.2$ for the lattice $16 \times 32^{3}$ and $\chi^{2} /$ dof $\simeq 4.5$ for the lattice $18 \times 44^{3}$.

- As was noted above the asymptotic behaviour of the spectral function at large $\omega$ is fixed $\rho \sim \omega^{4}$. However, the coefficient in front of the $\omega^{4}$-behaviour is modified by higher order radiative corrections (see formula (2.5)). In order to take this effect into account in formula (3.1) we introduced factor $A$. At this point our ansatz deviates from that used in papers $[10,11]$. If we take $A=1$, the ansatz (3.1) will not be able to describe our data $\left(\chi^{2} / \operatorname{dof}>100\right.$ for both lattices $)$.

- The values of the fit parameters are physically well motivated. For instance, the value of the parameter $A$ is smaller than unity, what agrees with the next-to-leading order result (2.5). The value of the strong coupling constant at the threshold parameter $\omega_{0}\left(\omega_{0} \sim 2.7 \mathrm{GeV}\right.$ in physical units) is $\alpha_{s}\left(\omega_{0}\right) \sim 0.2-0.3$. This allows us to expect that perturbative expression for the spectral function is applicable for $\omega>\omega_{0}$.

Low frequency part of the spectral function (3.1) is given by the first-order hydrodynamic expression $\sim \omega$. Comparison of the spectral functions of the energy-momentum tensor correlation functions obtained in N = 4 SYM [27] and the first-order hydrodynamic expressions allows us to expect that this approximation works well up to $\omega \leq \pi T \simeq 1 \mathrm{GeV}$ [28]. From the other side high frequency perturbative expression for the spectral function is fixed very accurately and it works well for $\omega \geq \omega_{0}=2.7 \mathrm{GeV}$. The form of the spectral function in the region $1 \mathrm{GeV} \leq \omega \leq 2.7 \mathrm{GeV}$ is not clear. Formula (3.1) continues the first-order hydrodynamic expression to the region $1 \mathrm{GeV} \leq \omega \leq 2.7 \mathrm{GeV}$ (see figure 3). As the result there is rather large discontinuity in the spectral function (3.1) at point $\omega_{0}$ which for the lattice $16 \times 32^{3}$ is $\left(\rho_{1}\left(\omega_{0}+0\right)-\rho_{1}\left(\omega_{0}-0\right)\right) / \rho_{1}\left(\omega_{0}-0\right) \simeq 6.7$. The last fact allows us to state that the spectral function in form (3.1) underestimates real spectral function in the region $1 \mathrm{GeV} \leq \omega \leq 2.7 \mathrm{GeV}$ and as a result the value of shear viscosity obtained in this fit is larger than its real value.

As was noted a serious drawback of the spectral function $\rho(\omega)$ is its discontinuity at point $\omega=\omega_{0}$. In order to solve this problem we propose another form of the spectral function which preserves basic ideas of the spectral function (3.1) and takes into account the property $\rho(-\omega)=-\rho(\omega)$

$$
\rho_{2}(\omega)=B T^{3} \omega+A \rho_{\mathrm{lat}}(\omega) \tanh ^{2}\left(\frac{\omega}{\omega_{0}}\right) .
$$

The fit of the lattice data $\left(x_{0} / a \geq 2\right)$ by the formula (2.2) with the spectral function (3.2) gives $A=0.723 \pm 0.003, B=0.039 \pm 0.012, \omega_{0} / T=5.6 \pm 0.6, \chi^{2} / d o f \simeq 1.2$ for the lattice $16 \times 32^{3}$ and $A=0.705 \pm 0.004, B=0.055 \pm 0.022, \omega_{0} / T=6.5 \pm 0.9, \chi^{2} / d o f \simeq 0.6$ for the lattice $18 \times 44^{3}$. It is seen that ansatz (3.2) for the spectral function fits the lattice data very well and parameters from different lattices are in a reasonable agreement with each other. The resulting shear viscosity is $\eta / s=0.088 \pm 0.027$ for the lattice $16 \times 32^{3}$ and $\eta / s=0.125 \pm 0.050$ for the lattice $18 \times 44^{3}$. In figure 3 we plot the spectral function $\rho_{2}(\omega)$ 


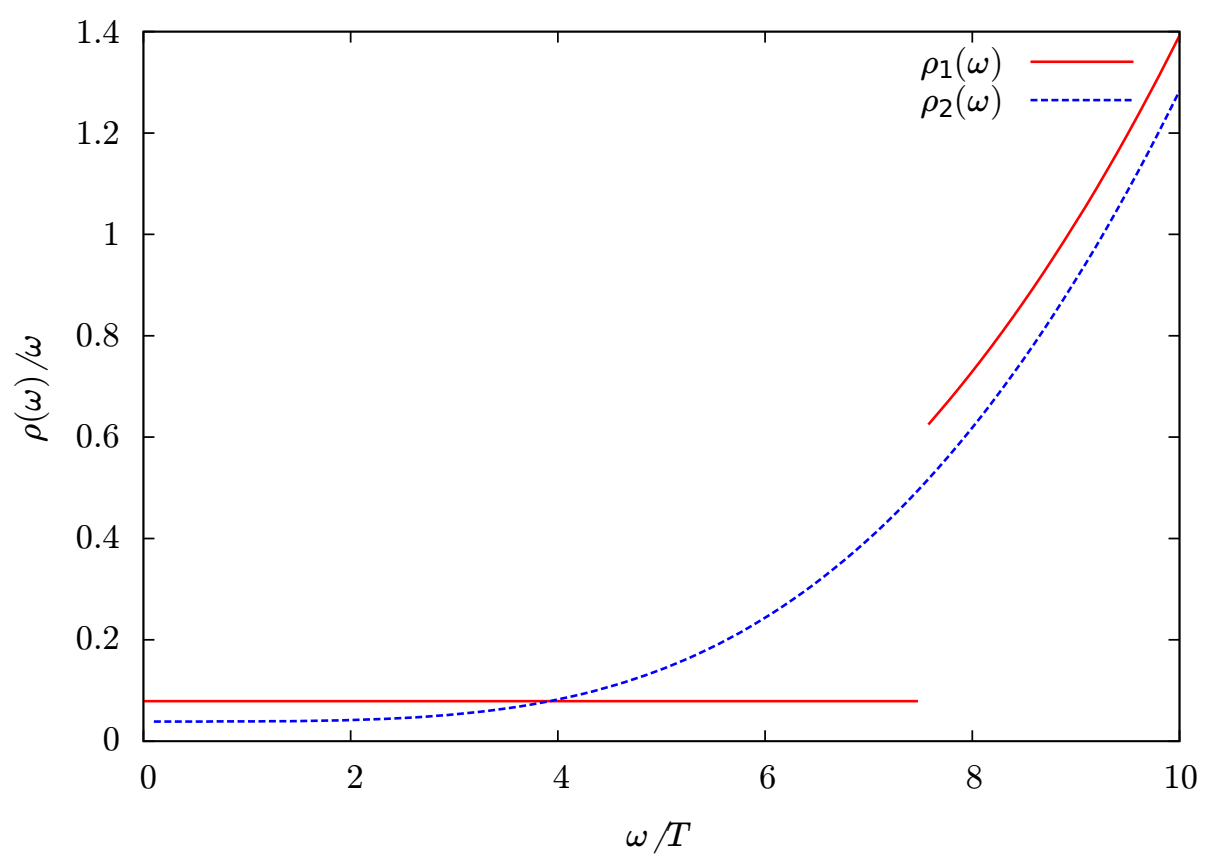

Figure 3. Spectral functions $\rho_{1}(\omega)$ and $\rho_{2}(\omega)$ as the function of $\omega / T$ for the lattice $L_{t}=16$.

as a function $\omega$ for the lattice $L_{t}=16$. Notice that the function (3.2) is larger than (3.1) in the region $1 \mathrm{GeV} \leq \omega \leq 2.7 \mathrm{GeV}$, as the result the value of the of shear viscosity becomes smaller. Note also that the value of shear viscosity obtained at the lattice $16 \times 32^{3}$ is very close to the $\mathrm{N}=4 \mathrm{SYM}$ prediction $\eta / s=1 / 4 \pi \simeq 0.080$.

In order to investigate the systematic errors, caused by the usage of the ansatz (8),(9) we studied other fit functions. Instead of the $\tanh ^{2}\left(\omega / \omega_{0}\right)$ in formula (3.2) one can use, for example, any power of this function $\sim \tanh ^{2 k}\left(\omega / \omega_{0}\right)$ or linear combination $\sim$ $\sum_{k} A_{k} \tanh ^{2 k}\left(\omega / \omega_{0}\right){ }^{2}$ We found, that in these cases the fits are also good and the resulting viscosities are greater than that for the spectral function (3.2) and smaller than that for the spectral function (3.1).

As the result of this paper we take the value

$$
\frac{\eta}{s}=0.134 \pm 0.034 \pm 0.046
$$

The central value is the average between the values of shear viscosity obtained with the spectral functions in the form (3.1) and (3.2) from the fit of lattice data measured at the lattice $16 \times 32^{3}$. The first uncertainty is due to the fitting procedure which is typically $25 \%$. The second uncertainty is due to the unknown model of the spectral function. We estimated this uncertainty assuming that the central value of shear viscosity can vary between central values of the fits (3.2) and (3.1). Actually there are a lot of different sources of uncertainties of our result but they are much smaller than statistical and spectral function model uncertainties.

\footnotetext{
${ }^{2}$ It should be noted here that in the region $1 \mathrm{GeV} \leq \omega \leq 2.7 \mathrm{GeV}$ any function can be approximated by this linear approximation with good accuracy.
} 


\section{Conclusion}

In this paper we studied the energy-momentum tensor correlation function $\left\langle T_{12}(0) T_{12}(x)\right\rangle$ for $\mathrm{SU}(2)$-gluodynamics using lattice simulation of QCD. We carried out the measurements of this correlation function at lattices $16 \times 32^{3}$ with $\beta=2.81$ and $18 \times 44^{3}$ with $\beta=2.85$. Both lattices correspond to deconfinement phase with the temperature $T / T_{c} \simeq 1.2$ but different physical volumes and lattice spacings. In order to enhance the accuracy of the calculation we used two-level algorithm which allowed us to reach accuracy $\sim 3 \%$ for the lattice $16 \times 32^{3}$ and $\sim 10 \%$ for the lattice $18 \times 44^{3}$ at point $x_{0}=1 / 2 T$. For the other points the accuracy is much better.

Using lattice data for the correlation function we tried to study the spectral function. It was shown that physically motivated anzatz which joins the first-order hydrodynamical behaviour at small frequencies with asymptotic freedom at large frequencies fits our data very well for both lattices. We also studied other forms of the spectral functions which interpolate between hydrodynamics and asymptotic freedom.

The results of the study of spectral functions allowed us to estimate the ratio of shear viscosity to the entropy density $\eta / s=0.134 \pm 0.057$. This value is in agreement with our previous finding $\eta / s=0.111 \pm 0.032[12]$ obtained at small lattice with the estimation of statistical uncertainty only. Note also that within the uncertainty of the calculation the value obtained in this paper agrees with the value measured in $\mathrm{SU}(3)$-gluodynamics: $\eta / s=0.102 \pm 0.056$ measured at temperature $T / T_{c}=1.24[10]$.

The values of the ratio $\eta / s$ for the $\mathrm{SU}(2)$ and $\mathrm{SU}(3)$-gluodynamics are very close to the prediction of the $\mathrm{N}=4 \mathrm{SYM}$ theory at strong coupling: $\eta / s=1 / 4 \pi$. So one can state that both theories belong to strongly correlated systems and probably the closeness of the ratio $\eta / s$ to the ratio in $\mathrm{N}=4 \mathrm{SYM}$ theory is the property on non-abelian gauge theories.

\section{Acknowledgments}

The simulations were performed at ITEP supercomputer. The work was supported by Far Eastern Federal University, Dynasty foundation, by RFBR grants 14-02-01185-a, 15-0207596-a, 15-32-21117 by a grant of the president of the RF, MD-3215.2014.2, and by a grant of the FAIR-Russia Research Center.

Open Access. This article is distributed under the terms of the Creative Commons Attribution License (CC-BY 4.0), which permits any use, distribution and reproduction in any medium, provided the original author(s) and source are credited.

\section{References}

[1] STAR collaboration, J. Adams et al., Experimental and theoretical challenges in the search for the quark gluon plasma: The STAR Collaboration's critical assessment of the evidence from RHIC collisions, Nucl. Phys. A 757 (2005) 102 [nucl-ex/0501009] [INSPIRE].

[2] PHENIX collaboration, K. Adcox et al., Formation of dense partonic matter in relativistic nucleus-nucleus collisions at RHIC: Experimental evaluation by the PHENIX collaboration, Nucl. Phys. A 757 (2005) 184 [nucl-ex/0410003] [INSPIRE]. 
[3] P.F. Kolb, P. Huovinen, U.W. Heinz and H. Heiselberg, Elliptic flow at SPS and RHIC: From kinetic transport to hydrodynamics, Phys. Lett. B 500 (2001) 232 [hep-ph/0012137] [INSPIRE].

[4] P. Huovinen, P.F. Kolb, U.W. Heinz, P.V. Ruuskanen and S.A. Voloshin, Radial and elliptic flow at RHIC: Further predictions, Phys. Lett. B 503 (2001) 58 [hep-ph/0101136] [inSPIRE].

[5] D. Teaney, J. Lauret and E.V. Shuryak, Flow at the SPS and RHIC as a quark gluon plasma signature, Phys. Rev. Lett. 86 (2001) 4783 [nucl-th/0011058] [INSPIRE].

[6] D.A. Teaney, Viscous Hydrodynamics and the Quark Gluon Plasma, arXiv:0905.2433 [INSPIRE].

[7] G. Policastro, D.T. Son and A.O. Starinets, The Shear viscosity of strongly coupled $N=4$ supersymmetric Yang-Mills plasma, Phys. Rev. Lett. 87 (2001) 081601 [hep-th/0104066] [INSPIRE].

[8] F. Karsch and H.W. Wyld, Thermal Green's Functions and Transport Coefficients on the Lattice, Phys. Rev. D 35 (1987) 2518 [InSPIRE].

[9] A. Nakamura and S. Sakai, Transport coefficients of gluon plasma, Phys. Rev. Lett. 94 (2005) 072305 [hep-lat/0406009] [INSPIRE].

[10] H.B. Meyer, A calculation of the shear viscosity in $\mathrm{SU}(3)$ gluodynamics, Phys. Rev. D 76 (2007) 101701 [arXiv:0704.1801] [INSPIRE].

[11] H.B. Meyer, Transport properties of the quark-gluon plasma from lattice QCD, Nucl. Phys. A 830 (2009) 641C [arXiv:0907.4095] [INSPIRE].

[12] V.V. Braguta and A.Yu. Kotov, Calculation of the viscosity of SU(2) gluodynamics with the lattice simulation, JETP Lett. 98 (2013) 127.

[13] R. Kubo, Statistical mechanical theory of irreversible processes. 1. General theory and simple applications in magnetic and conduction problems, J. Phys. Soc. Jap. 12 (1957) 570 [INSPIRE].

[14] H.B. Meyer, Locality and statistical error reduction on correlation functions, JHEP 01 (2003) 048 [hep-lat/0209145] [INSPIRE].

[15] H.B. Meyer, Energy-momentum tensor correlators and spectral functions, JHEP 08 (2008) 031 [arXiv:0806.3914] [INSPIRE].

[16] G. Aarts and J.M. Martinez Resco, Transport coefficients, spectral functions and the lattice, JHEP 04 (2002) 053 [hep-ph/0203177] [INSPIRE].

[17] A.L. Kataev, N.V. Krasnikov and A.A. Pivovarov, Two Loop Calculations for the Propagators of Gluonic Currents, Nucl. Phys. B 198 (1982) 508 [Erratum ibid. B 490 (1997) 505] [hep-ph/9612326] [INSPIRE].

[18] J. Engels, F. Karsch and T. Scheideler, Determination of anisotropy coefficients for SU(3) gauge actions from the integral and matching methods, Nucl. Phys. B 564 (2000) 303 [hep-lat/9905002] [INSPIRE].

[19] H.B. Meyer, Transport Properties of the quark-gluon Plasma: A Lattice QCD Perspective, Eur. Phys. J. A 47 (2011) 86 [arXiv:1104.3708] [INSPIRE].

[20] J. Engels, F. Karsch, H. Satz and I. Montvay, Gauge Field Thermodynamics for the SU(2) Yang-Mills System, Nucl. Phys. B 205 (1982) 545 [INSPIRE]. 
[21] H.B. Meyer, Finite temperature sum rules in lattice gauge theory, Nucl. Phys. B 795 (2008) 230 [arXiv:0711.0738] [INSPIRE].

[22] J. Engels, F. Karsch and K. Redlich, Scaling properties of the energy density in $\mathrm{SU}(2)$ lattice gauge theory, Nucl. Phys. B 435 (1995) 295 [hep-lat/9408009] [INSPIRE].

[23] A. Amato, G. Aarts, C. Allton, P. Giudice, S. Hands and J.-I. Skullerud, Electrical conductivity of the quark-gluon plasma across the deconfinement transition, Phys. Rev. Lett. 111 (2013) 172001 [arXiv:1307.6763] [INSPIRE].

[24] G. Aarts, C. Allton, A. Amato, P. Giudice, S. Hands and J.-I. Skullerud, Electrical conductivity and charge diffusion in thermal QCD from the lattice, JHEP 02 (2015) 186 [arXiv: 1412.6411] [INSPIRE].

[25] M.A. Shifman, A.I. Vainshtein and V.I. Zakharov, $Q C D$ and Resonance Physics. Theoretical Foundations, Nucl. Phys. B 147 (1979) 385 [InSPIRE].

[26] H.B. Meyer, Cutoff Effects on Energy-Momentum Tensor Correlators in Lattice Gauge Theory, JHEP 06 (2009) 077 [arXiv:0904.1806] [INSPIRE].

[27] P. Kovtun and A. Starinets, Thermal spectral functions of strongly coupled $N=4$ supersymmetric Yang-Mills theory, Phys. Rev. Lett. 96 (2006) 131601 [hep-th/0602059] [INSPIRE].

[28] H.B. Meyer, Energy-momentum tensor correlators and viscosity, PoS (LATTICE 2008) 017 [arXiv: 0809.5202] [INSPIRE]. 